población a los servicios de salud. Además, uno de los indicadores clave para la salud infantil es el grado de escolaridad de las madres y, en el Perú, la tasa general de alfabetización de la mujer es de $16 \%$. El Ministerio de Salud está trabajando para mejorar la situación de la AIEPI y recientemente se cuenta con el apoyo de nuevos asociados internacionales. Otros países que desean establecer una amplia cobertura equitativa de servicios de salud que favorezcan la supervivencia infantil pueden aprender de la experiencia del Perú. (Huicho L, et al. Scaling up Integrated Management of Childhood Illness to the national level: achievements and challenges in Peru. Health Policy Plan 2005;20(1):14-24.)

\section{Prevención del embarazo de adolescentes en una escuela secundaria de Chile}

En Chile, 15,6\% de los niños nacidos vivos entre 1996 y 1998 tenían madres de 15 a 19 años de edad. La tasa de embarazo en ese grupo de edad es de 40 por 1000 , excluidos los abortos ilegales, que no se registran. Durante el crecimiento físico y emocional de la adolescencia, las niñas a menudo se exponen a comportamientos sexuales que conllevan el riesgo de embarazos involuntarios e infecciones de transmisión sexual. Ambos casos se dan con frecuencia creciente tanto en Chile como en otros países. Las adolescentes no tienen acceso a anticonceptivos eficaces e interesa educarlas para que pospongan su experiencia sexual. Con ese fin, se evaluó el programa TeenSTAR, que se centra en la abstinencia, en una escuela pública de enseñanza secundaria para niñas en Santiago, Chile.

El grupo de estudio comprendió a 1259 jovencitas, que se distribuyeron en tres cohortes de acuerdo con el año (de 1996 a 1998) en que empezaron su educación secundaria. Las alumnas de la cohorte de 1996 no se sometieron a ninguna intervención. Las de 1997 y 1998 se distribuyeron aleatoriamente en dos grupos, con permiso escrito de los padres. En un grupo se probó el programa TeenSTAR y en el otro (grupo testigo) no se les ofreció instrucción alguna sobre el comportamiento sexual. El estudio fue prospectivo, con seguimiento de todas las cohortes durante los cuatro años de instrucción secundaria. Las tasas de embarazo de los grupos de intervención y de referencia se registraron y más tarde se contrastaron y se midieron. Se calculó la razón de riesgos (IC95\%) de cada grupo.

Las participantes eran jóvenes de raza blanca, pertenecían a familias con ingresos medianos o bajos y tenían de 15 a 16 años de edad. La intervención abarcó a 210 de las 423 alumnas de la cohorte de 1997 y a 328 de las 411 en la cohorte de 1998. El programa hizo hincapié en la importancia de los aspectos biológicos y fisiológicos de la fecundidad y los aspectos psicológicos y personales de la sexualidad. La instrucción se impartió en 14 unidades semanales durante un año escolar. Se presentaron como temas principales las diferencias entre los sexos; los prejuicios sociales al respecto; anatomía y fisiología de los órganos reproductivos; pubertad en ambos sexos; anotación de los períodos de fecundidad de la mujer; dominio de las emociones y del comportamiento; manipulación de la sexualidad en los medios; control y cumplimiento de decisiones; matrimonio y familia; comienzo y valor de la vida humana; métodos de planificación familiar; y embarazo, parto y lactancia. Cada unidad se compuso de períodos de discusión y lluvia de ideas; instrucción sobre la fecundidad; asignación de deberes; estudio de videos; y cultivo de la capacidad personal por medio de dramatización, actuación y debates en grupo. En la unidad 12 se les enseñaba la teoría de los métodos anticonceptivos, si bien no se recomendaba su uso, ya que el programa abogaba por la abstinencia. Las estudiantes podían participar voluntariamente en sesiones de orientación confidenciales. Se hizo hincapié sobre todo en estimular en cada adolescente el sentido de su propio valor y en promover su capacidad para tomar decisiones basadas en información confiable. Se capacitaron como monitores del programa maestras regulares de distintas materias, tuvieran o no relación con la educación sexual o la biología.

En la cohorte de 1996 (425 alumnas) se registraron 53 embarazos durante el período de seguimiento, con una tasa anual promedio de $3,86 \%$. En el grupo de intervención de la cohorte de 1997 (210 alumnas) hubo 6 embarazos durante el período de seguimiento y 35 en el grupo testigo (213 alumnas); es decir, las tasas fueron $3,3 \%$ y $18,9 \%$, respectivamente (riesgo relativo [RR]: 0,176; intervalo de confianza de 95\% [IC95\%]: 0,076-0,408). En la cohorte de 1998, hubo 13 embarazos en el grupo de intervención (328 alumnas) y 17 en el grupo testigo (83 alumnas), con tasas de $4,4 \%$ y $22,6 \%$, respectivamente (RR: 0,195; IC95\%: 0,099-0,384). El programa claramente se mostró eficaz para prevenir el embarazo no deseado en adolescentes y su influencia se extendió a los cuatro años de instrucción secundaria. La colaboración de las maestras que sirvieron de monitoras fue sumamente importante. Como resultado de esa experiencia, a partir de 1999 el programa TeenSTAR se ha incluido como parte del currículo regular de todas las estudiantes de primer año. (Cabezón $C$, et al. Adolescent pregnancy prevention: an abstinence-centered randomized controlled intervention in a Chilean public high school. J Adolesc Health 2005;36(1):64-9.) 